\title{
The effects of frequency and predictability on eye fixations in reading: An evaluation of the E-Z Reader model
}

\author{
Laurent Sparrow, Sébastien Miellet, and Yann Coello \\ UPRES URECA, Université de Lille 3, Villeneuve d'Ascq Cedex, France
}

\begin{abstract}
We tested whether the E-Z Reader model can be generalised to French language. The simulation showed that the model can account for the frequency effect. The predictability effect is moreover accurate for words skipping but not for fixation times. We think that this model is psychologically plausible for certain aspects of reading and we used it to evaluate the performance of dyslexic readers.
\end{abstract}

The E-Z Reader 7 model was applied to data that were obtained in an eye-tracking experiment. The purpose of the study was twofold. Its first aim was to test whether the model can be generalised to French language. The second objective was to test whether the model can satisfy to contextual constraint influencing reading, namely predictability. Fifteen participants were requested to read a French text that contained 134 words varying in frequency and predictability (the latter factor was determined in a previous task by asking participants to guess word $n+1$ when provided with the sentence up to word $n$ ). As in Reichle et al., the words were divided into five frequency classes and eye movement was recorded.. Furthermore, we ran a simulation to determine how well the model could predict the observed data (i.e., means duration and distributions of fixations). To be in agreement with E-Z Reader 7 , the first and last words of each sentence were not included in the data analysis.

As shown in Figure 1, we obtained a satisfactory matching between observed and predicted values for all dependent variables. E-Z Reader 7 correctly predicted the negative monotonic relationship between first fixation duration and word frequency. However, the predicted values were slightly smaller than the observed values for gaze duration. But gaze duration, which includes refixation, is considered a global indicator of word processing as it is influenced by postlexical processing, although first fixation is supposed to be more sensitive to low-level word processing of word. Contrary to the reference experiment previously used to test the model (Schilling et al., 1998), a crucial aspect of our study was that subjects read a meaningful text, which suggests that postlexical processing were more deeply probed. In the Schilling et al. study, subjects read sentences that tended to be short, stereotyped expressions that were very simple to comprehend; and even though reading was involved with this paradigm, it did not obviously draw on the kinds of complex comprehension processes that are part of full-blown reading. However, it is noteworthy that the E-Z Rreader model provides a theoretical framework for understanding the interface between vision and low-level aspects of language processing. 

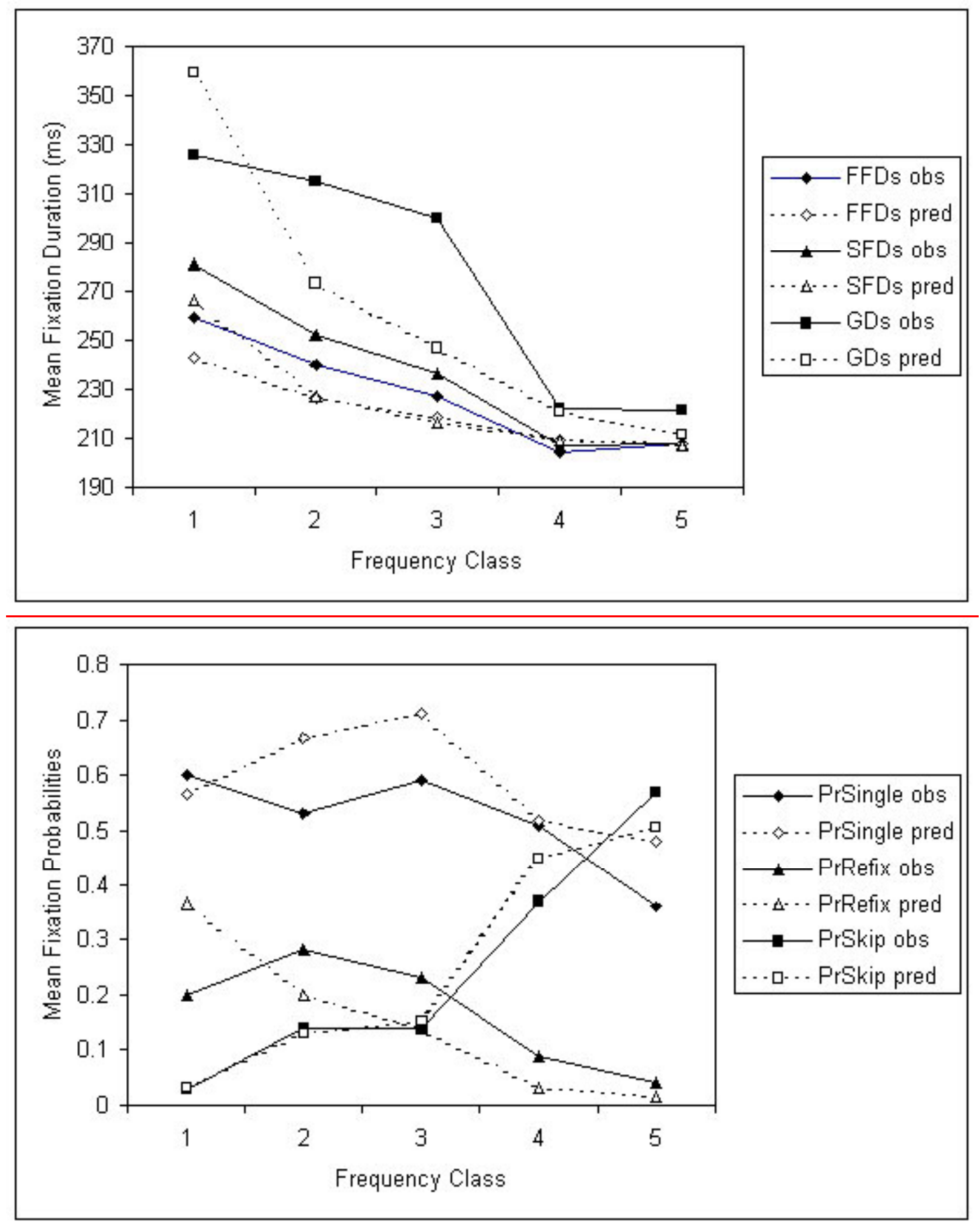

Figure 1: On top panel: mean observed (obs) and predicted (pred) first-fixation (FFD), singlefixation (SFD), and gaze duration (GD) for five frequency classes of words. The bottom panel shows the mean observed and predicted single-fixation (PrSingle), refixation (PrRefix), and skipping probabilities (PrSkip) for five frequency classes of words (RMSD=0.452). 
In addition to the frequency effect, an important finding was the predictability effect on eye movement. Generally, the effect of predictability is not as high as the frequency effect on fixation duration, but is pronounced on word skipping. In our study, readers skipped $31 \%$ of predictable words compared with $22 \%$ of the unpredictable words. The predictions of E-Z Reader were very close those values: $37 \%$ of predictable words and $25 \%$ of unpredictable words were skipped. The difference between predictable and unpredictable words ( $9 \%$ in our study and $12 \%$ for E-Z Reader) is quite consistent with prior research (Rayner et al. 2001; Rayner \& Well, 1996). However, inconsistency was observed in the pattern of results for fixation times (Table 1). E-Z Reader did not predict the effect of predictability on first fixation duration and single fixation. With respect to gaze duration, the effect predicted by EZ Reader was comparable in size to the effect obtained in other studies (Rayner et al. 2001; Rayner \& Well 1996), though it was lower than in our study.

\begin{tabular}{c|cc|cc|cc|} 
& \multicolumn{2}{|c|}{ FFDs } & \multicolumn{2}{c|}{ SFDs } & \multicolumn{2}{c|}{ GDs } \\
Predictability & Obs & pred & obs & pred & obs & pred \\
\hline Low & 226 & 213 & 232 & 212 & 280 & 240 \\
High & 207 & 209 & 212 & 209 & 235 & 223 \\
\hline Difference & 19 & 4 & 20 & 3 & 45 & 17
\end{tabular}

Table 1: Mean observed (obs) and predicted (pred) first-fixation (FFD), single-fixation (SFD), and gaze duration (GD), in milliseconds, for predictable and unpredictable words.

A closer examination of the data indicated that the prediction of E-Z -rReader was inconsistent for high frequency words: Fixation duration was longer for predictable than for unpredictable words (Table 2). For low-frequency words, the prediction of E-Z Reader appears to be larger for first and single fixations duration. However, the effect predicted by E$\mathrm{Z}$ Reader on gaze duration was comparable to the effect obtained in our study.

\begin{tabular}{c|cc|cc|cc|} 
& \multicolumn{2}{|c|}{ FFDs } & \multicolumn{2}{c|}{ SFDs } & \multicolumn{2}{c|}{ GDs } \\
& obs & pred & Obs & pred & obs & pred \\
\hline LF & 15 & 29 & 15 & 29 & 55 & 48 \\
HF & 22 & -23 & 24 & -23 & 35 & -14
\end{tabular}

Table 2: Effect of predictability for low frequency (LF) and high frequency (HF) words (in $\mathrm{ms})$.

This difference in the pattern of results for fixation duration versus word skipping can be accounted for by the different mechanisms that might be involved with regard to the decision about when and where moving the eye. With E-Z Reader, the time required to complete the first stage of lexical access (i.e., when to move the eye) is principally a function of word frequency and a free parameter (theta;, see Equation 1 in the target paper) reduces the extent to which the predictability of a word attenuates the lexical processing time. Our data suggests that, in normal reading, predictability can play a more important role during the first stage of lexical access (i.e., L1 ). 
Finally, the last version of E-Z Reader appears to be psychologically plausible and gives an accurate account of various phenomena in reading. It is, however, incomplete, as it takes preferentially into account "low-level" aspects of reading process. This model nonetheless provides a valuable analytical tool to examine some key assumptions about eye-movement and language processing. As an example, we used the model to simulate how individual differences would affect the pattern of eye movements in reading. For this purpose, we compared observed eye movements of dyslexic subjects with the E-Z Reader-predicted data. The observed and predicted values were very close for the duration of first fixation $(224 \mathrm{~ms}$ and $213 \mathrm{~ms}$ respectively for dyslexic subjects and E-Z Reader). However, gaze duration was considerably longer for dyslexics $(384 \mathrm{~ms})$ than for E-Z Reader $(256 \mathrm{~ms})$. This pattern of results can suggest that later stages of lexical access were impaired in dyslexic subjects but not the low-level aspect of reading process. Of course, further investigations are required to corroborate this conclusion.

\section{References}

Rayner, K., Binder K. S., Ashby, J., \& Pollatsek, A. (2001) Eye movement control in reading: Word predictability has little influence on initial landing positions in words. Vision Research 41:943-9-54. [LS]

Rayner, K. \& Well, A. D. (1996) Effects of contextual constraint on eye movements in reading: A further examination. Psychonomic Bulletin \& Review 3:504-09.

Schilling, H. E. H., Rayner, K., \& Chumbley, J. I. (1998) Comparing naming, lexical decision, and eye fixation times: Word frequency effects and individual differences. Memory and Cognition 26:1,270-81. 